\title{
Percutaneous Dilational Tracheotomy for Airway Management in a Newborn With Pierre-Robin Syndrome and a Glossopharyngeal Web
}

\author{
Arash Pirat MD, Selim Candan MD, Aytekin Ünlükaplan MD, Özgür Kömürcü MD, \\ Selim Kuşlu MD, and Gülnaz Arslan MD
}

\begin{abstract}
Pierre-Robin syndrome (PRS) is often associated with difficulty in endotracheal intubation. We present the use of percutaneous dilational tracheotomy (PDT) for airway management of a newborn with PRS and a glossopharyngeal web. A 2-day-old term newborn with PRS and severe obstructive dyspnea was evaluated by the anesthesiology team for airway management. A direct laryngoscopy revealed a glossopharyngeal web extending from the base of the tongue to the posterior pharyngeal wall. The infant was spontaneously breathing through a $2 \mathrm{~mm}$ diameter fistula in the center of this web. It was decided that endotracheal intubation was impossible, and a PDT was planned. The trachea of the newborn was cannulated, using a 20 gauge peripheral venous catheter and a $0.71 \mathrm{~mm}$ guide wire was introduced through this catheter. Using 5 French, 7 French, 9 French, and 11 French central venous catheter kit dilators, staged tracheotomy stoma dilation was performed. By inserting a size 3.0 tracheotomy cannula, PDT was successfully completed in this newborn. This case describes the successful use of PDT for emergency airway management of a newborn with PRS and glossopharyngeal web. Key words: difficult airway management; difficult endotracheal intubation; percutaneous tracheotomy. [Respir Care 2012;57(4):627-630. ( 2012 Daedalus Enterprises]
\end{abstract}

\section{Introduction}

Pierre-Robin syndrome (PRS) is a rare congenital craniofacial anomaly characterized by glossoptosis, micrognathia, and retrognathia. ${ }^{1}$ Children with PRS may present with airway obstruction, which, without appropriate respiratory management, can result in death. ${ }^{2}$ Evans and coworkers ${ }^{3}$ have reported that in a series of 115 patients with PRS the incidence of airway problems that required an

\footnotetext{
The authors are affiliated with the Department of Anesthesiology, Baskent University Faculty of Medicine, Ankara, Turkey.

Dr Pirat presented a version of this paper at the 40th Critical Care Congress of the Society of Critical Care Medicine, held January 15-19, 2011, in San Diego, California.

The authors have disclosed no conflicts of interest.

Correspondence: Arash Pirat MD, Department of Anesthesiology, Baskent University, 10 Sok No 45, Bahcelievler Ankara 06490, Turkey. E-mail: araspirat@gmail.com.
}

DOI: $10.4187 /$ respcare.01404 intervention was $44 \%$. In another retrospective study of 110 children with PRS, prone posturing alone effectively relieved the mild airway obstruction in 82 patients; however, in 28 infants with severe respiratory distress, endotracheal intubation was required. ${ }^{4}$ Recently, Leboulanger and others have demonstrated that noninvasive ventilation may also be considered as an effective treatment for improving breathing pattern and respiratory outcomes in patients with PRS. ${ }^{5}$

Despite the fact that conventional airway management techniques are usually effective for airway control in most patients with PRS, facial abnormalities that accompany PRS may lead to difficulties in both mask ventilation and endotracheal intubation in a subset of these patients. ${ }^{1}$ Previously, several techniques have been described for management of difficult airway in patients with PRS. $.^{6-8} \mathrm{How}-$ ever, to the best of our knowledge there are no reports on the use of percutaneous dilational tracheotomy (PDT) for airway management of patients with PRS. PDT is an established technique for airway management in adult critically ill patients who require prolonged mechanical ventilation. Several studies have shown that PDT is quicker, 


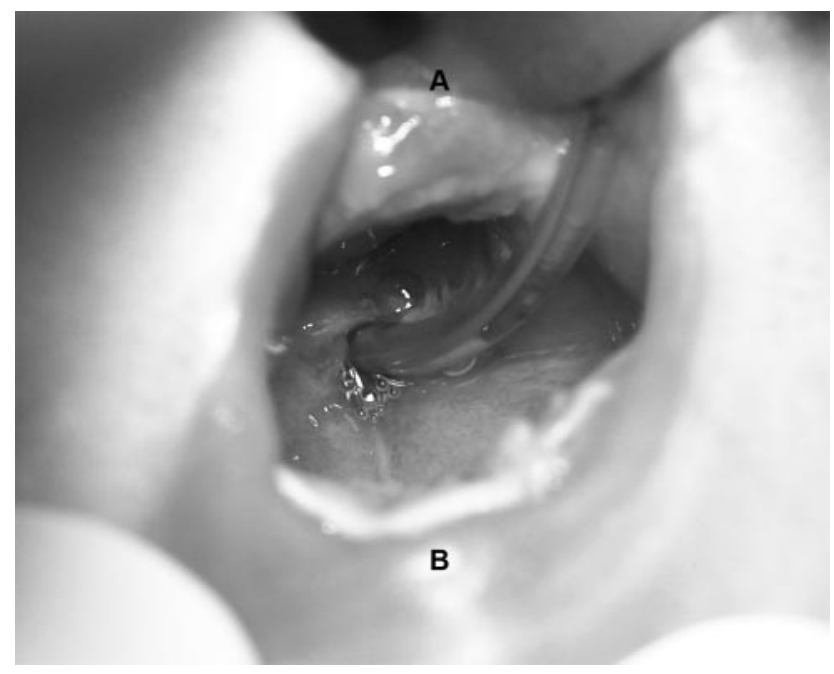

Fig. 1. Glossopharyngeal web extending from the base of the tongue (A) to the posterior pharyngeal wall (B). An 8 French catheter is inserted through the fistula opening in the middle of the web.

more cost-effective, and more convenient than surgical tracheotomy in ICU patients. ${ }^{9-11}$ However, cervical injury, pediatric age, coagulopathy, and emergency airway access are considered as contraindications to PDT. ${ }^{11,12}$

We present a newborn with PRS and airway obstruction due to glossopharyngeal web who was successfully treated with PDT for emergency airway management.

\section{Case Report}

A 2-day-old term newborn female (weight $3.1 \mathrm{~kg}$, height $49 \mathrm{~cm}$ ) with PRS was admitted to the neonatal intensive care unit (NICU) for progressive respiratory distress from a maternity hospital at midnight. At 3:00 Am the anesthesiology team was consulted for airway management of the newborn, after unsuccessful intubation attempts by the NICU team. The infant had severe dyspnea, with subcostal and suprasternal retractions. She was receiving $2 \mathrm{~L} / \mathrm{min}$ of oxygen through nasal cannula, and her oxygen saturation was $85-90 \%$. A direct laryngoscopy revealed a rudimentary tongue and a web extending from the base of the tongue to the posterior pharyngeal wall. She was spontaneously breathing through a $2 \mathrm{~mm}$ diameter fistula in the center of this mass (Fig. 1). An attempt was made to pass a stylet through the opening of the fistula to perform a blinded endotracheal intubation. However, it seemed that the fistula had a tortuous shape and it was not possible to thread the stylet through it.

At this point it was decided that endotracheal intubation was impossible and the ear, nose, and throat team was consulted for an emergency surgical tracheotomy. However, considering the patient's airway problem and her severe respiratory distress, the on call junior ear, nose, and throat physician expressed his unwillingness to perform a surgical tracheotomy before obtaining a secure airway. A PDT was planned. After midline subcutaneous infiltration of $0.5 \mathrm{~mL}$ of $1 \%$ lidocaine at the upper tracheal level, the trachea of the newborn was cannulated using a 20 gauge peripheral venous catheter. The puncture site was $0.5 \mathrm{~cm}$ below the level of the cricoid cartilage, and successful tracheal cannulation was confirmed by aspirating air through the catheter into a $2 \mathrm{~mL}$ syringe. As the next step, a 5 French central venous catheter kit's $0.71 \mathrm{~mm}$ guide wire was introduced through the peripheral venous catheter and a $0.5 \mathrm{~cm}$ horizontal incision was made, centering the guide wire. The central venous kit's 5 French dilator was then advanced over the guide wire to dilate the subcutaneous tissue and anterior tracheal wall. After this first stage dilation, the dilator was removed and a 14 gauge peripheral venous catheter was inserted in the trachea over the guide wire. At this point the guide wire was exchanged with a 7 French central venous catheter kit's $0.81 \mathrm{~mm}$ guide wire. Second, third, and fourth stage dilations were performed using 7 French, 9 French, and 11 French dilators, respectively. Finally, the 7 French dilator was used as the inner cannula of a size $3 \mathrm{~mm}$ tracheotomy cannula, and the tracheotomy tube and dilator were inserted into the trachea over the guide wire. Bilateral equal and good chest movements were observed, and her obstructive dyspnea resolved immediately. We did not use any sedating or paralyzing agents throughout the procedure in this patient because of the unusual airway anatomy and high risk of respiratory arrest.

The next day she was taken to the operating room for excision of the glossopharyngeal web and revision of the tracheotomy by the pediatric surgery and ear, nose, and throat teams, respectively. A gastrostomy was inserted for enteral feeding 1 week later. Two months later the patient underwent horizontal mandibular osteotomies, with multivector external distractor placement.

One month after mandibular osteotomies her tracheotomy cannula was removed in the pediatric ICU. However, after tracheal decannulation she developed severe obstructive dyspnea immediately. Although several attempts were made to reinsert a tracheotomy cannula through the stoma, the tracheotomy tract was lost and these attempts were unsuccessful. Because the glossopharyngeal web was excised, it was presumed that upper airway patency had been established, and endotracheal intubation was attempted. However, endotracheal intubation attempts using different laryngoscope blades and blinded intubation were unsuccessful. In order to perform intubation or achieve ventilation via a laryngeal mask airway, 2 attempts were made to insert size 1.0 and 1.5 laryngeal mask airways. However, it was not possible to properly insert a laryngeal mask airway and ventilate the patient. She had inspiratory stridor and her oxygen saturation was $88 \%$ with $2 \mathrm{~L} / \mathrm{min}$ of 
nasal oxygen. While preparing the fiberoptic intubation equipment, the patient's dyspnea was worsening and it was decided to proceed with retrograde intubation. After cleansing the skin with povidone-iodine, the trachea was fixed between the thumb and the index finger of the left hand. Under local anesthesia, the trachea was cannulated through the cricothyroid membrane with an 18 gauge peripheral venous catheter, in the cephalic direction. A $0.81 \mathrm{~mm}$ central venous catheter guidewire was passed through the needle into the pharynx, and it was retrieved through the mouth under direct laryngoscopy, using a Magill forceps. The wire was then used to guide a size $3.5 \mathrm{~mm}$ endotracheal tube through the vocal cords. Bilateral equal and good chest movements were observed, and the patient was moved to the operating room for a surgical tracheotomy. Her hospital stay was uneventful afterwards, and she was discharged home 10 days later with a tracheotomy and gastrostomy ( 3 mo and $20 \mathrm{~d}$ old). Her tracheotomy cannula was removed when she was 10 months old.

\section{Discussion}

The craniofacial abnormalities that accompany PRS may lead to difficult airway management. ${ }^{1,13}$ Previous reports have described several techniques for airway management in patients with PRS. 2,6-8,14-16 Nevertheless, during our literature review we could not find any report on the use of PDT for airway management in patients with PRS. The use of PDT for emergency airway management in a newborn is highly controversial. Traditionally, both pediatric age and emergency airway necessity are accepted as contraindications for PDT. ${ }^{10,12}$ However, the contraindications of PDT have gradually reduced, and its indications have expanded over time. Several reports have demonstrated the safety and feasibility of PDT in patients with the above contraindications. ${ }^{17-19}$ Ben-Nun et al ${ }^{17}$ have reported the successful use of a modified Griggs technique for emergency PDT in 10 adult trauma patients.

Despite promising preliminary reports, there are several technical obstacles to performing PDT in pediatric patients. ${ }^{18,19}$ The trachea and larynx are smaller and more mobile and compliant in children. Furthermore, even minimal external compression can totally collapse the tracheal lumen in infants. ${ }^{18,19}$ Therefore, there is very limited experience with PDT in children, and PDT associated complications may be more frequent in children than adults. ${ }^{19}$ Nevertheless, successful use of PDT in children and even infants have been reported previously. ${ }^{18,19}$

It may be argued that the next step for airway management in this patient should have been a surgical tracheotomy or a needle cricothyroidotomy. However, because of the reluctance of the surgical team, a surgical tracheotomy could not be performed. A needle cricothyroidotomy was not chosen for airway management in this patient because a permanent airway was needed. The traditional approach is to use needle cricothyroidotomy as a short-term (usually less than $45 \mathrm{~min}$ ) temporizing airway measure because of the concern for carbon dioxide retention. ${ }^{20}$ There are no commercially available PDT kits for children; therefore, we used central venous catheter kits to perform PDT in this newborn. Although far from perfect, in experienced hands, this method is simple and the required equipment is widely accessible.

Retrograde intubation has been used for management of difficult tracheal intubation for almost 50 years. ${ }^{21}$ This technique has been recommended in the difficult airway management algorithm of several national anesthesiology societies. ${ }^{21}$ Unfortunately, retrograde intubation is rarely taught or practiced, and has been reported as an underused intubation technique in the management of the difficult airway. ${ }^{21}$ In our patient, even after excision of the glossopharyngeal web and corrective mandibular osteotomies, it was not possible to perform an endotracheal intubation or insert a laryngeal mask airway, as described above. Because imminent cardiac arrest due to hypoxemia was ensuing, we decided to proceed with a retrograde intubation instead of waiting for the fiberoptic intubation equipment to arrive. In our practice and that of others, the time required to perform a retrograde intubation is short and this technique can be life-saving. ${ }^{21}$

In conclusion, this case demonstrates that, for newborns in whom endotracheal intubation is impossible and surgical tracheotomy is not available, PDT may be considered as a rescue technique for emergency airway management.

\section{REFERENCES}

1. Nargozian $\mathrm{C}$. The airway in patients with craniofacial abnormalities. Paediatr Anaesth 2004;14(1):53-59.

2. Horikiri M, Park S, Kinoshita M, Matsumoto D. Respiratory management of Pierre Robin sequence using nasopharyngeal airway with Kirschner wire. J Plast Reconstr Aesthet Surg 2010;63(4):e390-e394.

3. Evans AK, Rahbar R, Rogers GF, Mulliken JB, Volk MS. Robin sequence: a retrospective review of 115 patients. Int J Pediatr Otorhinolaryngol 2006;70(6):973-980.

4. Li HY, Lo LJ, Chen KS, Wong KS, Chang KP. Robin sequence: review of treatment modalities for airway obstruction in 110 cases. Int J Pediatr Otorhinolaryngol 2002;65(1):45-51.

5. Leboulanger N, Picard A, Soupre V, Aubertin G, Denoyelle F, Galliani E, et al. Physiologic and clinical benefits of noninvasive ventilation in infants with Pierre Robin sequence. Pediatrics 2010;126(5): e1056-e1063.

6. Portnoy JE, Tatum S. Retrograde nasal intubation via the cleft in Pierre-Robin Sequence neonates: a case series. Int J Pediatr Otorhinolaryngol 2009;73(12):1828-1832.

7. Smallman B, Ball R, Tatum S. A novel technique of retrograde nasal intubation for the Pierre Robin Sequence infant with a known difficult airway. Paediatr Anaesth 2009;19(9):919-921.

8. Vlatten A, Aucoin S, Gray A, Soder C. Difficult airway management with the STORZ video laryngoscope in a child with Robin Sequence. Paediatr Anaesth 2009;19(7):700-701. 


\section{Tracheotomy in a Newborn With Pierre-Robin Syndrome and a Glossopharyngeal Web}

9. Pirat A, Zeyneloglu P, Candan S, Akkuzu B, Arslan G. Percutaneous dilational tracheotomy in solid-organ transplant recipients. Transplant Proc 2004;36(1):221-223.

10. Delaney A, Bagshaw SM, Nalos M. Percutaneous dilatational tracheostomy versus surgical tracheostomy in critically ill patients: a systematic review and meta-analysis. Crit Care 2006; 10(2):R55.

11. Freeman BD, Isabella K, Lin N, Buchman TG. A meta-analysis of prospective trials comparing percutaneous and surgical tracheostomy in critically ill patients. Chest 2000;118(5):1412-1418.

12. Oliver ER, Gist A, Gillespie MB. Percutaneous versus surgical tracheotomy: an updated meta-analysis. Laryngoscope 2007;117(9): 1570-1575.

13. Barak M, Capdevila M, Katz Y. Fatal airway obstruction from percutaneous endoscopic gastrostomy in an infant with Pierre Robin sequence. Anesth Analg 2007;105(1):292-293.

14. Asai T, Nagata A, Shingu K. Awake tracheal intubation through the laryngeal mask in neonates with upper airway obstruction. Paediatr Anaesth 2008;18(1):77-80.
15. Cruz MJ, Kerschner JE, Beste DJ, Conley SF. Pierre Robin sequences: secondary respiratory difficulties and intrinsic feeding abnormalities. Laryngoscope 1999;109(10):1632-1636.

16. Johnson SE, Tatum SA, Thomson LL. Pierre Robin sequence in a patient with ectrodactyly-ectodermal dysplasia-clefting syndrome: a case report and review of the literature. Int J Pediatr Otorhinolaryngol 2002;66(3):309-313.

17. Ben-Nun A, Altman E, Best LA. Emergency percutaneous tracheostomy in trauma patients: an early experience. Ann Thorac Surg 2004;77(3):1045-1047.

18. Fantoni A, Ripamonti D. [Tracheostomy in pediatrics patients]. Minerva Anestesiol 2002;68(5):433-442. Article in Italian.

19. Principi T, Morrison GC, Matsui DM, Speechley KN, Seabrook JA, Singh RN, et al. Elective tracheostomy in mechanically ventilated children in Canada. Intensive Care Med 2008;34(8):1498-1502.

20. Mace SE, Khan N. Needle cricothyrotomy. Emerg Med Clin North Am 2008;26(4):1085-1101.

21. Dhara SS. Retrograde tracheal intubation. Anaesthesia 2009;64(10): 1094-1104. 